\title{
The atomization current and droplet size of ethanol in two different small-scale electro-spraying systems
}

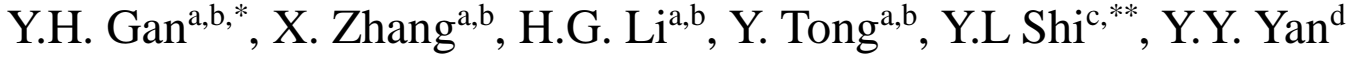 \\ a School of Electric Power, South China University of Technology, Guangzhou, \\ 510640, China; \\ ${ }^{\mathrm{b}}$ Guangdong Province Engineering Research Center of High Efficient and Low \\ Pollution Energy Conversion, Guangzhou, 510640, China; \\ ${ }^{c}$ Teaching and Training Center for Engineering Basis, South China Agricultural \\ University, Guangzhou 510640, China; \\ ${ }^{\mathrm{d}}$ Fluids \& Thermal Engineering Group, Faculty of Engineering, The University of \\ Nottingham, University Park, Nottingham NG7 2RD, UK;
}

\section{Submitted for publication in Journal of Electrostatics}

*Corresponding author: Prof. Yunhua Gan, Ph.D.,

Address: School of Electric Power, South China University of Technology, Wushan Road, Tianhe District, Guangzhou 510640, China;

Tel/Fax: 00-86-20-87110613;

Email: ganyh@scut.edu.cn.

**Corresponding author: Ms.Yanling Shi,

Address: Teaching and Training Center for Engineering Basis, South China

Agricultural University, Wushan Road, Tianhe District, Guangzhou 510640, China;

Tel/Fax: 00-86-20-85281443;

Email: yanlingsally@scau.edu.cn. 


\section{Abstract}

2 An experimental study on electro-spraying from small-scale combustors is carried out

3 using liquid ethanol as fuel. Two systems of electro-spraying are employed in the

4 present study; one is a nozzle system (without a ring electrode) and the other is a

5 nozzle-ring system (with a ring electrode). The photos of electro-spraying at the

6 cone-jet mode are taken by a digital camera. The voltage drop across the resistance in

7 the loop is measured by a data acquisition instrument, and the atomization current is

8 calculated according to Ohm's Law. The size and velocity of electro-spraying droplets

9 are measured by a Phase Doppler Anemometer. A non-dimensional analysis on atomization current is completed to explain the electro-spraying phenomena of liquid at the stable cone-jet mode. The results show that, the lower atomization current and droplet velocity corresponds to smaller size of droplet. Based on the results of non-dimensional analysis, it is found that the dimensionless atomization current in both the nozzle system and nozzle-ring system obeys the scaling law as square root of the dimensionless flow rate. The charge density is of a -1.5 power dependence on droplet diameter. Both of the nozzle and the nozzle-ring systems show a good agreement with Rayleigh instability.

Keywords: cone-jet mode; electro-spraying; atomization current; droplet size; droplet velocity; non-dimensional analysis 


\section{Introduction}

With the progress in micro-fabrication techniques, there is an increasing demanding for the miniaturization of mechanical and electro-mechanical engineering devices. The miniaturized power generating devices using liquid hydrocarbon as fuel with high specific energy may have more competitive advantages than those using batteries [1-2]. Zeleny photographed drops held at the end of capillary tubes and raised to a high potential, forming a jet of glycerine from an electrified drop [3]. Since then, many experiments and simulations have been carried to study this phenomenon [4-9]. A conical meniscus is formed at the tip of the nozzle, and followed by a ligament, the narrow jet broken into monodisperse droplets due to Rayleigh instability. No droplets coalescence will take place due to the coulombic forces generated by the electric field; this mode of electrospray is known as "cone-jet" mode [10]. Thong and Weinberg [11] used the electric fields for dispersing solid and liquid fuels, and this made it possible that the droplet size and the charge in terms of the parameters of applied electrical, geometrical and flow can be predicted.

The cone-jet is a stable atomization mode. Many researchers have carried out experimental investigations on the stability of the cone-jet mode and the dependence on the liquid properties, flow rate and the electrostatic conditions of the current and droplet size [12-15]. Fernandez and Loscertales [13] found that the scaling law of the spray current emits from an electrified meniscus and fits an equation based on the square root of flow rate of the highly conducting liquid. Gañan-Calvo et al. [14] found another different dependence between the current and flow rate for the liquid of low 
1 polarity. In fact, an atomization process is affected by many parameters. In order to

2 propose a general dimensional description of the entire range of working parameter

3 for cone-jet electrospraying, a two-dimensional parametrical 'chart' with four distinct

4 regions and corresponding scaling laws for droplet size and current was established by

5 Gañan-Calvo [15].

6 According to the scaling laws, very low flow rate is needed to produce small

7 droplets. A low flow-induction charger was used to improve aerosol delivery

8 efficiency [16]. The formation of a stable Taylor cone was very important for

9 electrospray operation. A ballpoint pen electrospray emitter greatly expands the operation range in the flow rate-voltage space [17]. A ring electrode was used to

11 prevent the Taylor cone frequently change its shape under various external disturbance [18-19]. There were many extraordinary properties of the cone-jet mode, such as monodispersity of the primary droplets; high charge on the surface of the generated droplets; controllable droplet size by varying the flow rate [12]. It remained necessary to identify whether the cone-jet spraying mode was obtained or not. The classifications are mainly based on visual observations of the liquid meniscus, but it is very hard to observe the morphology of the spraying in the practical application.

Some experiments were done to research the relationship between the current and the behavior of the liquid meniscus [20-21]. Verdoold et al. [22] found a general mapping between the properties of the current through the system and the spraying mode that was independent of the material properties of the liquid, the electrode geometry and other experimental conditions. 

compared to investigate the effect of the ring electrode on the cone-jet characteristics.

3 Numerical calculation was performed to investigate the effect of ring electrode on the

4 electric field. The same electro-spraying systems are employed in the present study.

5 The combustor in nozzle-ring system is consisted of three pieces of quartz glass tube

6 connected by a ring electrode and a stainless steel grid electrode. Because of the

7 reflections on the quartz tubes, the spray modes are difficult to be observed clearly,

8 and the photos of electro-spraying can only be obtained after removing the outer

9 surface of the combustors [19]. Although the size and velocity distributions of electro-spraying droplets could not be measured by a Phase Doppler Anemometer through the quartz tubes, the atomization current could be measured. Therefore, to obtain the relationship between the atomization current and droplet size is very important. In the present study, the photos of electro-spraying at the cone-jet mode are obtained by a digital camera. The voltage drop across the resistance between the grid and the ground is measured by a data acquisition instrument, and the atomization current is calculated according to Ohm's law. The size and velocity distributions of electro-spraying droplets are measured by a Phase Doppler Anemometer both for the nozzle system (without a ring electrode) and the nozzle-ring system (with a ring electrode). A non-dimensional analysis on atomization current is proposed to explain the electro-spraying phenomena of liquid at the stable cone-jet mode. 


\section{Experimental setup}

\subsection{The test rig}

The test rig is shown in Fig.1, which consists of a liquid fuel feeding system, a test section, a high voltage supply system. A capillary is used as a nozzle, which is supported by the substrate (a ceramic package). The fuel is supplied though a plastic tube to the nozzle by a syringe pump (KDS100, KD SCIENTIFIC, USA) with $\pm 1.0 \%$ accurancy. The test section consists of a fuel-supply nozzle, a ring electrode (only for the nozzle-ring system) and a ground electrode (a stainless steel grid).

Three types' diagnostic techniques are employed to monitor the electro-spraying modes. They are (1) the observations, in which the photographs of different electro-spraying modes are taken by a digital single-lens reflex camera (Cannon EOS 5D Mark III) with a green laser light as an illuminating light source; (2) the size and velocity distributions measurements, of which the size and velocity distributions of electro-spraying droplets are measured by a Phase Doppler Anemometer (Particle Dynamics Analysis, Dantec, Denmark); (3) the current measurements, in which the voltage drop across the resistance between the grid and the ground is measured by a data acquisition instrument, and the atomization current is calculated according to Ohm's law.

The liquid fuel used is pure ethanol $\left(\mathrm{CH}_{3} \mathrm{CH}_{2} \mathrm{OH}\right.$, molecular weight of 46.07 , purity $>99.5 \%$ ). A conductivity meter with $\pm 1.0 \%$ accuracy (Rex; DDS-307A; Shanghai, China) is applied for measuring the conductivity of ethanol. 


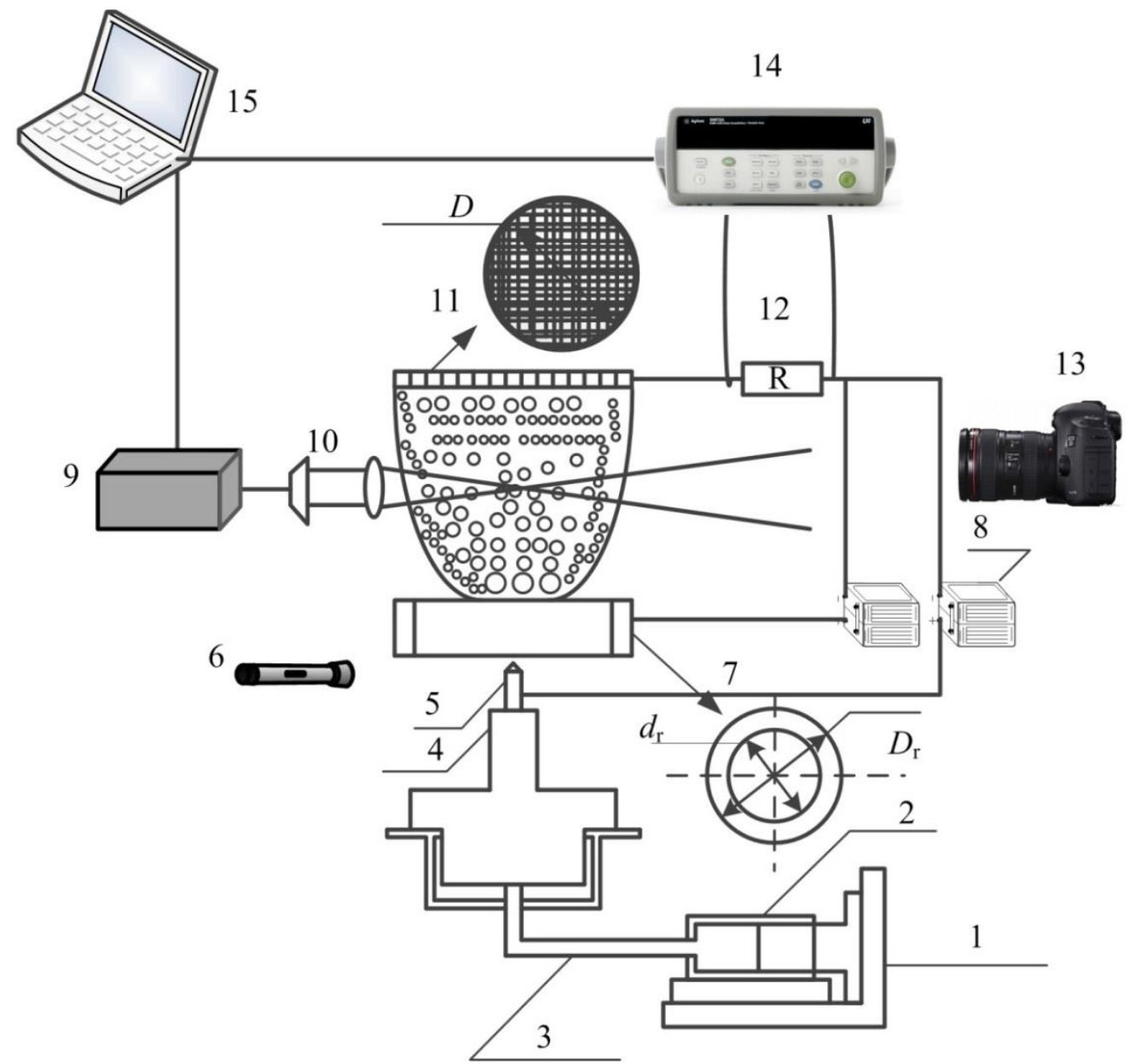

2 1- syringe pump; 2- syringe; 3- plastic pipe; 4- substrate; 5- nozzle; 6-laser lamp; 7-

3 ring electrode; 8- high-voltage DC power source; 9- laser; 10-focusing lens;

4 11-steelgrid; 12- resistance; 13- digital single-lens reflex camera; 14- data acquisition instrument; 15-computer

Fig.1 Schematic diagram of experimental setup

\section{2 test section}

The two electro-spraying systems are employed in the present experiment, namely, the nozzle system in which the nozzle is maintained at high potential by connecting it to a direct-current (DC) power source (71030P, GENVOLT, UK), and a stainless steel grid is grounded; and the nozzle-ring system in which the nozzle and the ring electrode are connected to two DC power source (71030P, GENVOLT, UK) 
1 separately, and the stainless steel grid is also grounded. The distance between the

2 nozzle and the stainless steel grid is the same for both the nozzle system and 3 nozzle-ring system. Fig.2 shows the test section and the distribution of measuring 4 cross-sections ( $\mathrm{z}=5.0 \mathrm{~mm}, 10.0 \mathrm{~mm})$ and points (No. 0- No. 16).

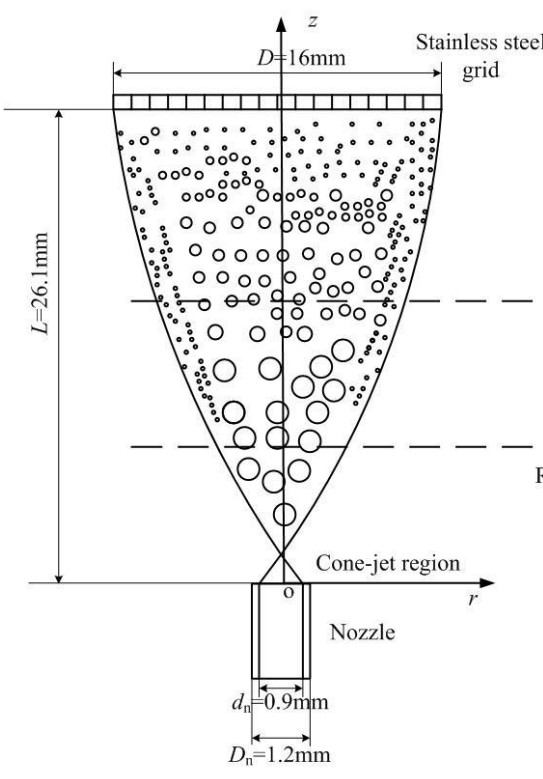

(a)

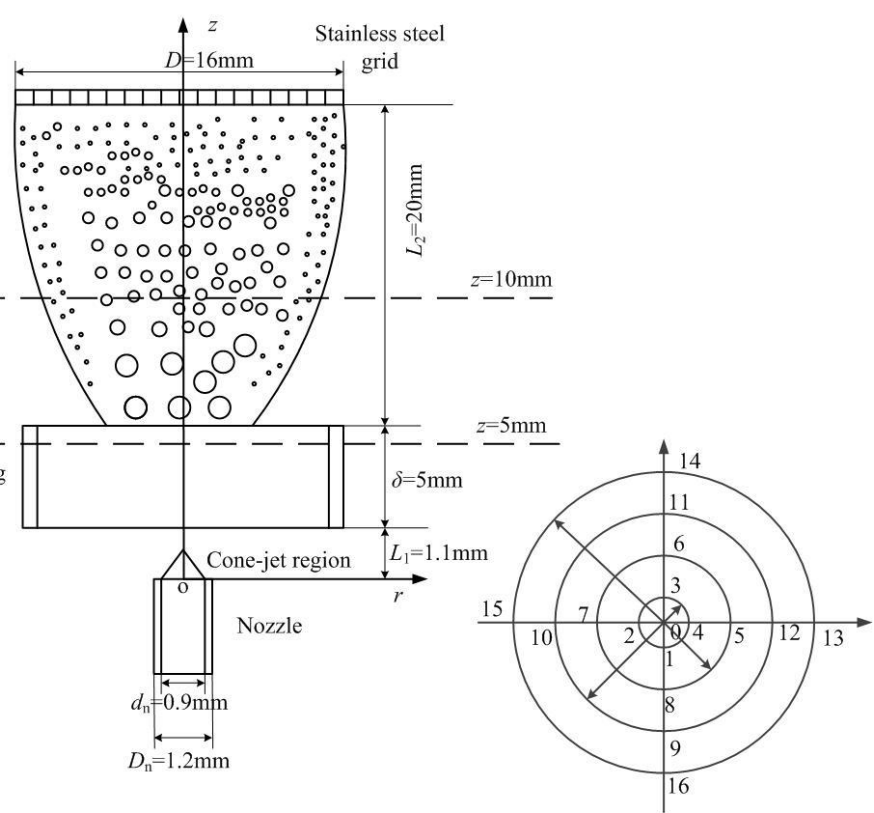

(b)

(c)

Fig.2 Test section and the distribution of measuring cross-sections and positions [19]

( a: test section of nozzle system; b: test section of nozzle-ring system; c: test points of a certain cross-section $(z=5.0 \mathrm{~mm}$ or $10.0 \mathrm{~mm}))$

The inner diameter of the stainless steel nozzle is $0.90 \mathrm{~mm}\left(d_{\mathrm{n}}=0.90 \mathrm{~mm}\right)$; the outer diameter, $1.20 \mathrm{~mm}\left(D_{\mathrm{n}}=1.20 \mathrm{~mm}\right)$; the inner diameter of the ring electrode, $12.40 \mathrm{~mm}\left(d_{\mathrm{r}}=12.40 \mathrm{~mm}\right)$; the outer diameter, $16.00 \mathrm{~mm}\left(D_{\mathrm{r}}=16.00 \mathrm{~mm}\right)$; and the thickness, $5.00 \mathrm{~mm}(\delta=5.00 \mathrm{~mm})$. The stainless steel grid has a diameter of $16.00 \mathrm{~mm}$, and each hole in it has a diameter of $1.00 \mathrm{~mm}$. A high DC power source (71030P, GENVOLT, UK) is used to supply high voltage on the nozzle $\left(V_{\mathrm{n}}\right)$. For the nozzle 
1 system, the stainless steel grid is arranged above the tip of the nozzle with a vertical

2 distance of $26.10 \mathrm{~mm}(L=26.10 \mathrm{~mm})$. For the nozzle-ring system, the ring electrode is

3 arranged above the tip of the nozzle with a distance of $1.10 \mathrm{~mm}\left(L_{1}=1.10 \mathrm{~mm}\right)$, and

4 the grid is arranged above the tip of the ring electrode with a vertical distance of 20.00

$5 \mathrm{~mm}\left(L_{2}=20.00 \mathrm{~mm}\right)$. Another DC power source (71030P, GENVOLT, UK) is

6 employed to supply high voltage on the ring electrode $\left(V_{\mathrm{r}}\right)$.

\subsection{Measuring system}

The spray current was so small that it is difficult to be measured directly by the electrometer. A standard $1 \mathrm{M} \Omega$ resistance is connected between the grid and the ground electrode. The electric potential difference across the resistance is measured, and the spray current is calculated by the measured potential. The signals are transferred to a computer though the data acquisition instrument (34790A, Agilent USA). The equivalent electrical circuit with spray is shown in Fig.3, which consists of DC power sources, electro-spray, electrodes, and the standard resistance. $V_{\mathrm{n}}$ is the voltage on nozzle; $V_{\mathrm{r}}$ is the voltage on ring electrode; $R_{1}$ is the total spray resistance in the nozzle system, $U_{1}$ is the voltage of $R_{1} ; R_{2}$ is the liquid cone-jet resistance, $U_{2}$ is the voltage of $R_{2} ; R_{3}$ is the spray resistance in the nozzle-ring system, $U_{3}$ is the voltage of $R_{3} ; R_{\mathrm{S}}$ is the standard resistance connected, $U_{\mathrm{s}}$ is the voltage of $R_{\mathrm{s}} ; I$ is the effective value of the spray current. According to Ohm's law, the electric current can be calculated.

$$
I=U_{s} / R_{s}
$$

The electric potential difference across the resistance is $0.00-0.08 \mathrm{~V}$, the power 


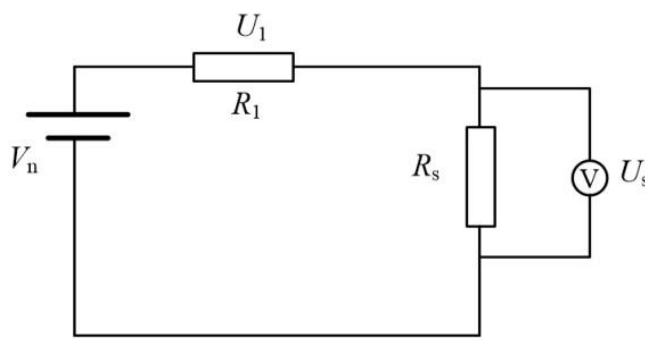
effect on the spray current can be ignored.

source voltage is $0.00-7.00 \mathrm{kV}$. Compared with the spray resistance, the standard 1 $\mathrm{M} \Omega$ resistance connected between the grid and the ground electrode is very low, its

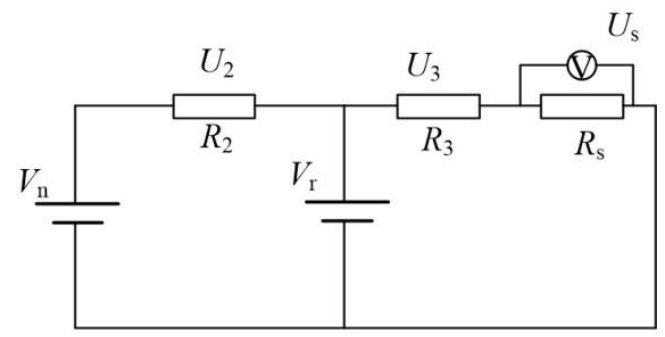

\section{4}

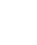

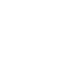

7

8

(s)

(1)

$$
\text { connected standard resistance; } U_{1}, U_{2}, U_{3}, U_{\mathrm{s}} \text { are the voltages across each resistances }
$$
(a) nozzle system
(b) nozzle-ring system

Fig.3 The equivalent electrical circuit with spray

velocity distributions of electro-spraying droplets. The PDA system includes a fiber optic probe, a signal processor, a receiver probe and an argon-ion laser. The sample number at each measuring position is set to 2000 and sampling time is 10 seconds, which can ensure the estimation of droplet size and velocity statistics to be accurate. The distribution of measuring sections and points is shown in Fig.2. The measurements of droplet sizes are made at different cross-sections $(\mathrm{z}=5.0 \mathrm{~mm}, \mathrm{z}=10.0$ $\mathrm{mm}$ ) up the tip of the nozzle. The measuring points distribute on the concentric circles of different radiuses $(r=0.5 \mathrm{~mm}, r=1.0 \mathrm{~mm}, r=1.5 \mathrm{~mm}$ and $r=2.0 \mathrm{~mm}$ ). After 
1

2

3 distribution is determined based on 17 points.

4

5

6

7 $8 \quad$ are from the handbook [23].

9

10

11

12

13

14

15

\subsection{Error summary}

Table 1 Physical properties of ethanol $\left(25^{\circ} \mathrm{C}\right)$ [19]

8

(1)

(1)

1

comparing the droplet size distribution at the different cross-sections, measurements are performed at the cross-section $(z=10.0 \mathrm{~mm})$ up the tip of the nozzle, and the size

In the present study, ethanol is used as liquid fuel and its physical properties at $25^{\circ} \mathrm{C}$ are shown in Table 1 . The conductivity of ethanol is measured by a conductivity meter with $\pm 1.0 \%$ uncertainty (Rex; DDS-307A; Shanghai, China). Other parameters

\begin{tabular}{lllll}
\hline $\begin{array}{l}\text { Density } \\
\mathrm{kg} / \mathrm{m}^{3}\end{array}$ & Viscosity & $\begin{array}{l}\text { Surface tension } \\
\mathrm{Pa} \cdot \mathrm{s}\end{array}$ & $\begin{array}{l}\text { Conductivity } \\
\mathrm{N} / \mathrm{m}\end{array}$ & $\begin{array}{l}\text { Relative permittivity } \\
\mathrm{S} / \mathrm{m}\end{array}$ \\
\hline 789.3 & $1.07 \times 10^{-3}$ & 0.022 & $5.1 \times 10^{-5}$ & 25.3 \\
\hline
\end{tabular}

.

The experiments are conducted in the following variation ranges of operating parameters: liquid ethanol flow rate $Q$ of $0.20-4.00 \mathrm{ml} / \mathrm{h}$, the applied voltage on the nozzle electrode $V_{\mathrm{n}}$ of $0.00-6.80 \mathrm{kV}$, the applied voltage on the ring electrode $V_{\mathrm{r}}$ of $1.00 \mathrm{kV}$, the distance $L_{1}$ of $1.10 \mathrm{~mm}$, the distance $L_{2}$ of $20.00 \mathrm{~mm}$, the distance $L$ of $26.10 \mathrm{~mm}$. Table 2 shows the measurement errors. 
Table 2 error summary

\begin{tabular}{llcc}
\hline Parameters & Measure tool or & Ranges & Error \\
& equipment & & \\
\hline Flow rate, $Q$ & Syringe pump & $0.00-4.00 \mathrm{ml} / \mathrm{h}$ & $\pm 1.0 \%$ \\
Voltage, $V_{\mathrm{n}}$ & DC power source & $0.00-2.00 \mathrm{kV}$ & $\pm 1.0 \%$ \\
Voltage, $V_{\mathrm{r}}$ & DC power source & $0.00-7.00 \mathrm{kV}$ & $\pm 1.0 \%$ \\
Voltage, $U \mathrm{~s}$ & Data acquisition & $0.00-10.00 \mathrm{mV}$ & $\pm 1.0 \%$ \\
& instrument & & \\
Diameter, & Vernier caliper & $0.90-16.00 \mathrm{~mm}$ & $\pm 0.02 \mathrm{~mm}$ \\
$d_{\mathrm{n}}, D_{\mathrm{n}}, d_{\mathrm{r}}, D_{\mathrm{r}}, D$ & & & \\
Thickness of ring & Vernier caliper & $5.00 \mathrm{~mm}$ & $\pm 0.02 \mathrm{~mm}$ \\
electrode, $\delta$ & & & $\pm 1.0 \%$ \\
Droplet size & PDA & $0.00-250.00 \mu \mathrm{m}$ & $\pm 1.0 \%$ \\
Droplet velocity & PDA & $0.00-50.00 \mathrm{~m} / \mathrm{s}$ & \\
\hline
\end{tabular}

2

3 3. Results and discussions

$4 \quad 3.1$ The atomization current at different modes

Fig.4 shows the influence of the nozzle voltage $V_{\mathrm{n}}$ on atomization current in the nozzle system. The distance between the stainless steel grid and the nozzle tip is of $L$ $=26.10 \mathrm{~mm}$. Fig. 5 shows the influence of nozzle voltage $V_{\mathrm{n}}$ on atomization current in the nozzle-ring system. The distance between the nozzle tip and the ring electrode is of $L_{1}=1.10 \mathrm{~mm}$, the distance between the ring electrode and the stainless steel grid is of $L_{2}=20.00 \mathrm{~mm}$, and the applied voltage on the ring electrode is of $V_{\mathrm{r}}=1.00 \mathrm{kV}$.

When the liquid drop is charged, the drop turns into a cone, then a liquid jet appears at the cone apex, and the jet is broken into micro-scale droplets due to Rayleigh instability. The charge is trapped on their surface, and the continuous motion 
1 of charged jet and droplets are formed atomization current. The conduction charging

2 is a process, in which the high voltage is connected to the liquid. The induction

3 charging is also a process, in which the high voltage electrode keeps a distance away

4 from the nozzle tip and the charge is induced on the liquid. The liquid droplets are

5 positively or negatively charged depending on the polarity of the high voltage supply.

6 For the nozzle electro-spraying system (in Fig.4), the droplets are charged mainly

7 based on conduction charging method. For the nozzle-ring electro-spraying system (in

8 Fig.5) the droplets are charged based on both the conduction charging and the

$9 \quad$ induction charging methods.

10

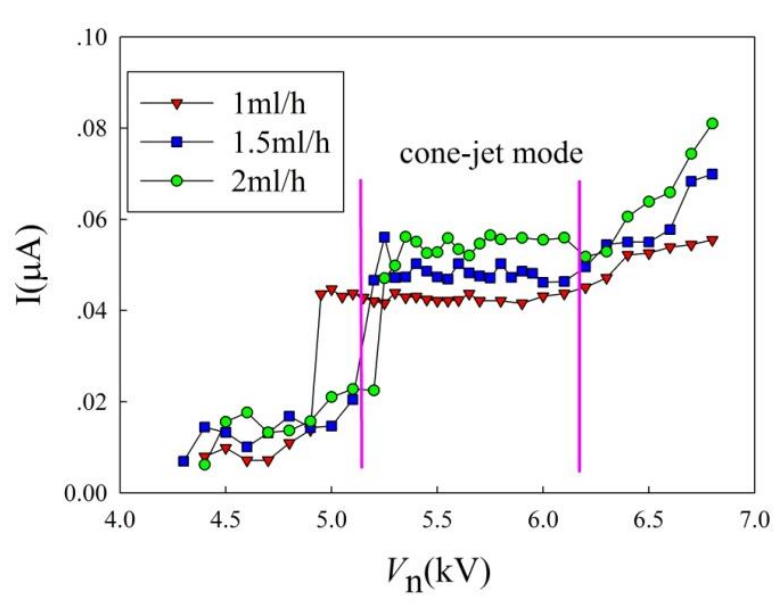

(a)

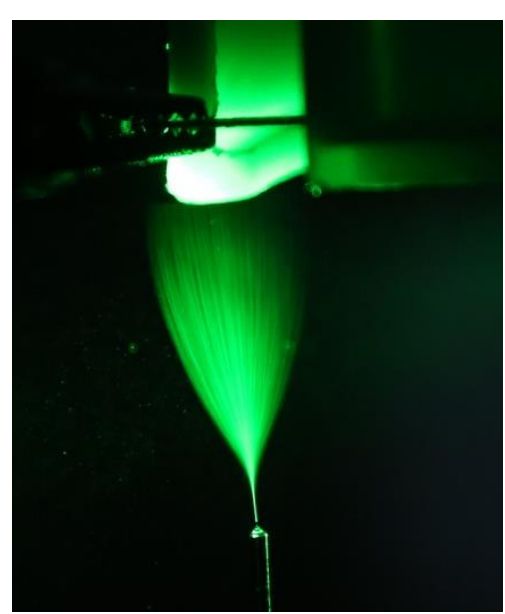

(b) 


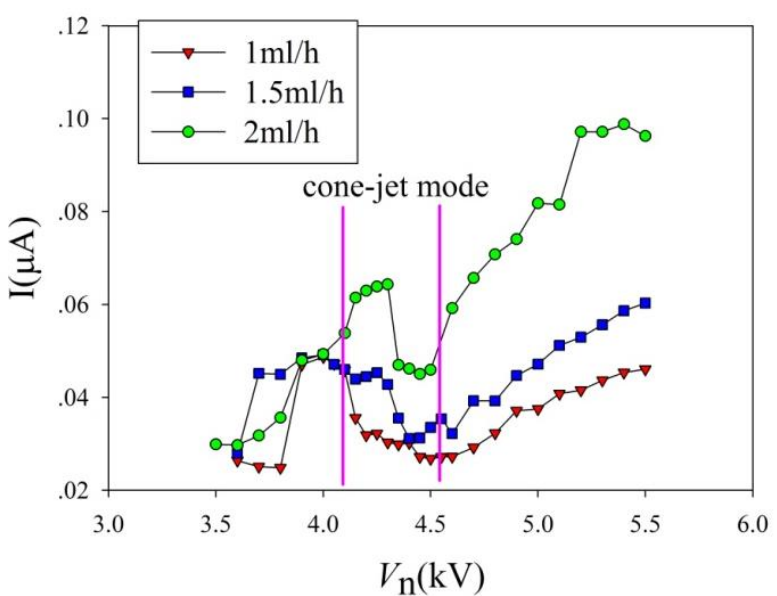

(a)

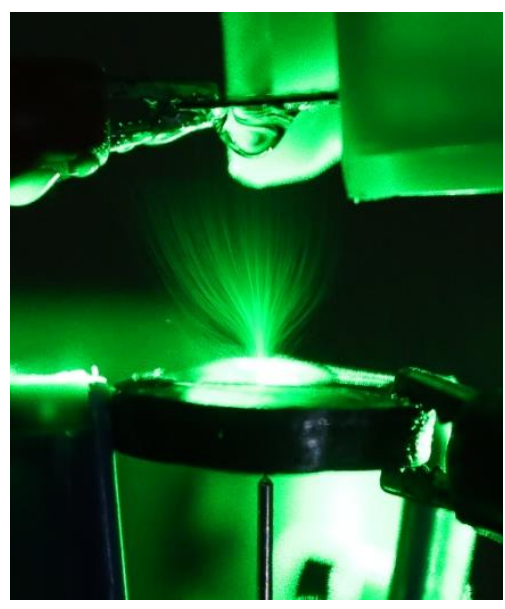

(b)

Fig.5 (a) Current-voltage characteristics for different spraying modes of nozzle-ring system $\left(L_{1}=1.10 \mathrm{~mm}, L_{2}=20.00 \mathrm{~mm}, V_{\mathrm{r}}=1.00 \mathrm{kV}\right)$; (b) a typical electrospray photo at cone-jet mode $\left(Q=1.00 \mathrm{ml} / \mathrm{h}, V_{\mathrm{n}}=4.40 \mathrm{kV}, V_{\mathrm{r}}=1.00 \mathrm{kV}\right)$

Corona discharge is a process, in which a current flows from an electrode with high potential into a neutral fluid, such as air, by ionizing the fluid so as to create a region of plasma around the electrode. The corona-generated space charges are driven by the electric field to the ground or to the opposite polarity causing the enhancement of the space electric field; and the total electric field could be called as ionized fields. The drifted space charges can cause the ion current in space and corona current on the conductor, respectively.

The empirical solution for a corona initiation field $E_{\mathrm{co}}(\mathrm{V} / \mathrm{m})$ from ring electrode of radius $r(\mathrm{~m})$ can be represented [25]:

$$
r>100 \mu \mathrm{m}
$$

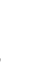

$$
E_{\text {co }}=3 \times 10^{6}\left(1+\frac{0.03}{r^{0.5}}\right)
$$

The radius of the ring electrode is $6.20 \mathrm{~mm}(r=6.20 \mathrm{~mm}), E_{\mathrm{co}}=3 \times 10^{6} \mathrm{~V} / \mathrm{m}$. 
2 flow rate of ethanol. Firstly, the current increases with electric potential $\left(V_{\mathrm{n}}=4.25\right.$

$3-5.10 \mathrm{kV})$. Then, it keeps almost constant at cone-jet mode $\left(V_{\mathrm{n}}=5.10-6.20 \mathrm{kV}\right)$. When

4 the electric potential further increases to a certain value (near to $6.20 \mathrm{kV}$ ), the electric

5 field intensity exceeds to the value of $E_{\mathrm{co}}$, the corona discharge is emerged, the air is ionized, and the current increases rapidly.

Fig.5 shows the variation of atomization current along with different nozzle electric potentials at a fixed ring electric potential of $1.00 \mathrm{kV}$ in nozzle-ring system. The combined electric field is established by the nozzle, the ring and the grid. The residence time of liquid ethanol plays a great role in the charging process. Both conduction charging and induction charging controls the electrospray process together. For conduction charging, there is a current flowing through the power supply, which causes electrical energy consumption; but there is no electrical energy consumption during the induction process. In the induction process, the liquid droplets are positively or negatively charged depending on the polarity of the high voltage power source. The ring electrode is connected to the positive of the DC power source. When the nozzle potential $V_{\mathrm{n}}$ is low $\left(V_{\mathrm{n}}=3.50-4.20 \mathrm{kV}\right)$, the axial velocity of liquid jet and droplet is relatively low, so the current increases with the nozzle potential $V_{\mathrm{n}}$. Then, it keeps almost constant at the initial stage of cone-jet mode $\left(V_{\mathrm{n}}=4.20-4.30 \mathrm{kV}\right)$. When the electric potential $V_{\mathrm{n}}$ is increased to a certain value $\left(V_{\mathrm{n}} \approx 4.3 \mathrm{kV}\right)$, the corona condition appears during the conduction and induction charging process. The air will be ionized to some extent, which causes neutralization with the charged droplets. 
1 Thus, the current decreases with the increase of the nozzle potential. When the nozzle

2 potential is increased to over $4.60 \mathrm{kV}$, the corona charging dominates the charging

3 process, the neutral air can be ionized greatly and the current is increased rapidly.

\subsection{The droplet size and velocity distributions at cone-jet mode}

The volume mean diameter is calculated from the mean of the droplet volumes as:

$$
D_{30}=\left\{\frac{1}{N} \sum_{i=1}^{N_{i}} n_{i} D_{i}^{3}\right\}^{\frac{1}{3}}
$$

where, $D_{\mathrm{i}}$ is the volume mean diameter of the individual particles, $N_{\mathrm{i}}$ the number of size classes (bins) selected by the user, $n_{\mathrm{i}}$ the number of particles in each size class and $N$ the total number of particles.

Fig. 6 shows the droplet size distributions under cone-jet mode at the different cross-sections. The distribution is very similar when measuring points is at $z=5 \mathrm{~mm}$ and $z=10 \mathrm{~mm}$, respectively. It is necessary to keep the measurements at a certain position to compare the two different systems. Considering the differences of two apparatus, the measurements performed at $z=10.0 \mathrm{~mm}$ is chosen to identify the difference between the two systems.

Fig.7 shows droplet size versus flow rate at cone-jet mode for two electrospray systems. Fig.8 shows droplet velocity versus flow rate at cone-jet mode for two electrospray systems. The droplet size and velocity distributions at cone-jet mode are uniform at different position (please see the measurement points No.0-No.16, Fig.2), so that the droplet size measured is as nearly monodisperse and the droplet velocity is 


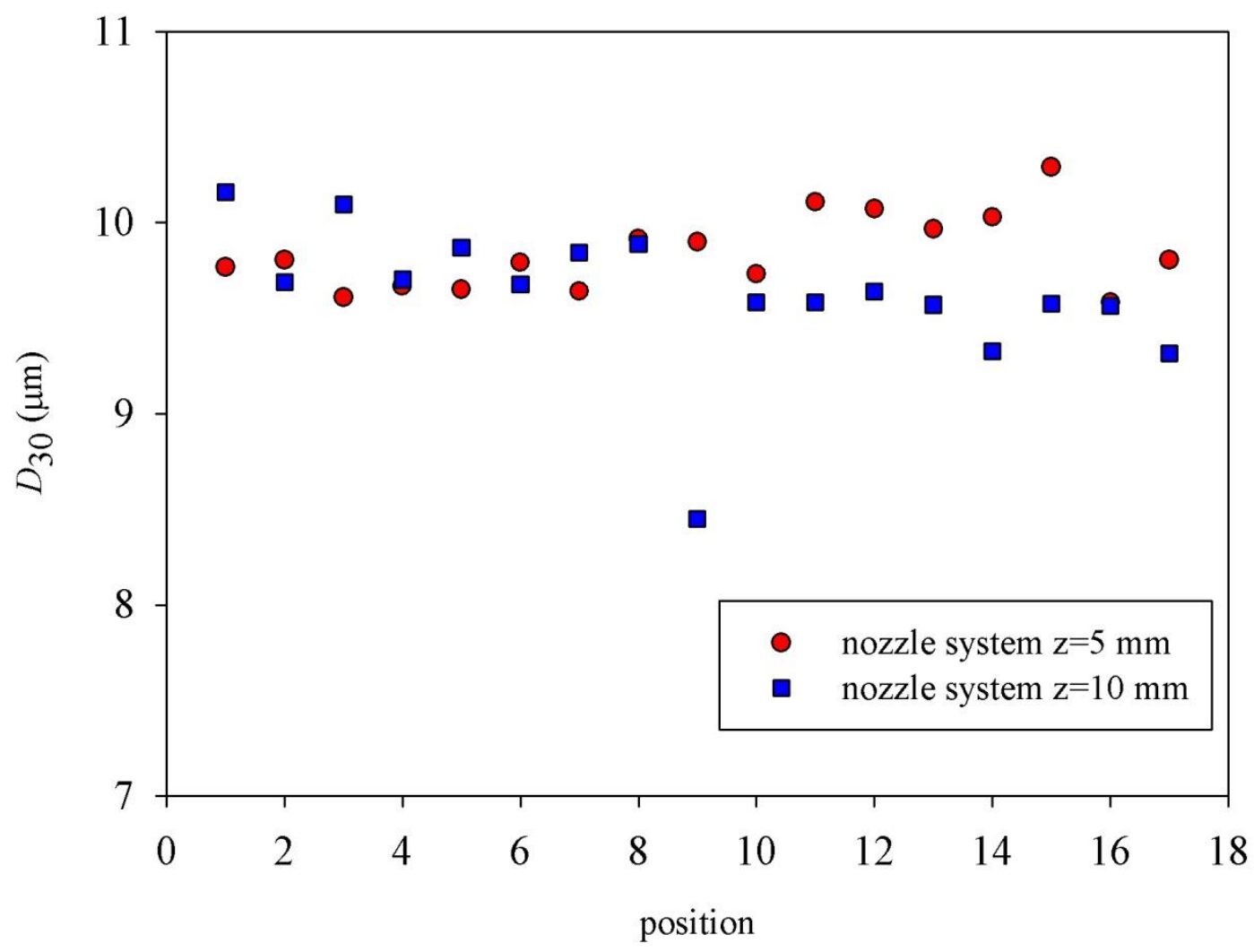

Fig.6 The distribution of measuring points under cone-jet mode at different crosssections $(z=5 \mathrm{~mm}, 10 \mathrm{~mm})$ for nozzle system $\left(V_{\mathrm{n}}=5.44 \mathrm{kV}, Q=1.00 \mathrm{ml} / \mathrm{h}\right)$

Fig. 7 shows that, for both nozzle and nozzle-ring systems, droplet size increases with flow rate. As shown in Fig.8, droplet velocity increases with flow rate. Thus, the droplet size and velocity increase with the flow rate; the lower velocity corresponds to smaller droplet size.

The velocity in the nozzle-ring system is smaller than that in the nozzle system, and the variation of the velocity is smaller in the nozzle-ring system. The droplets breakup process is dominated by the axisymmetric disturbance. For the nozzle-ring system, the axisymmetric disturbance is protected by the ring electrode, due to the 
1 decreasing of the electric field strength near the cone-tip. The electric field strength in

2 the nozzle-ring system is smaller than that in the nozzle system at the axial position

$3(\mathrm{z}=10.0 \mathrm{~mm})[19]$. At small flow rate $(0.40 \mathrm{ml} / \mathrm{h}<Q<2.00 \mathrm{ml} / \mathrm{h})$, the liquid is charged

4 completely, and the droplets have more time to evaporate due to its smaller droplet

5 velocity, so the droplet size is smaller in the nozzle-ring system. At large flow rate

$6 \quad(2.00 \mathrm{ml} / \mathrm{h}<Q<3.20 \mathrm{ml} / \mathrm{h})$, the liquid is charged incompletely. In addition, the electric

7 field strength in the nozzle-ring system is smaller than that in the nozzle system. Thus,

8 the droplet size becomes larger than that in the nozzle system.

9

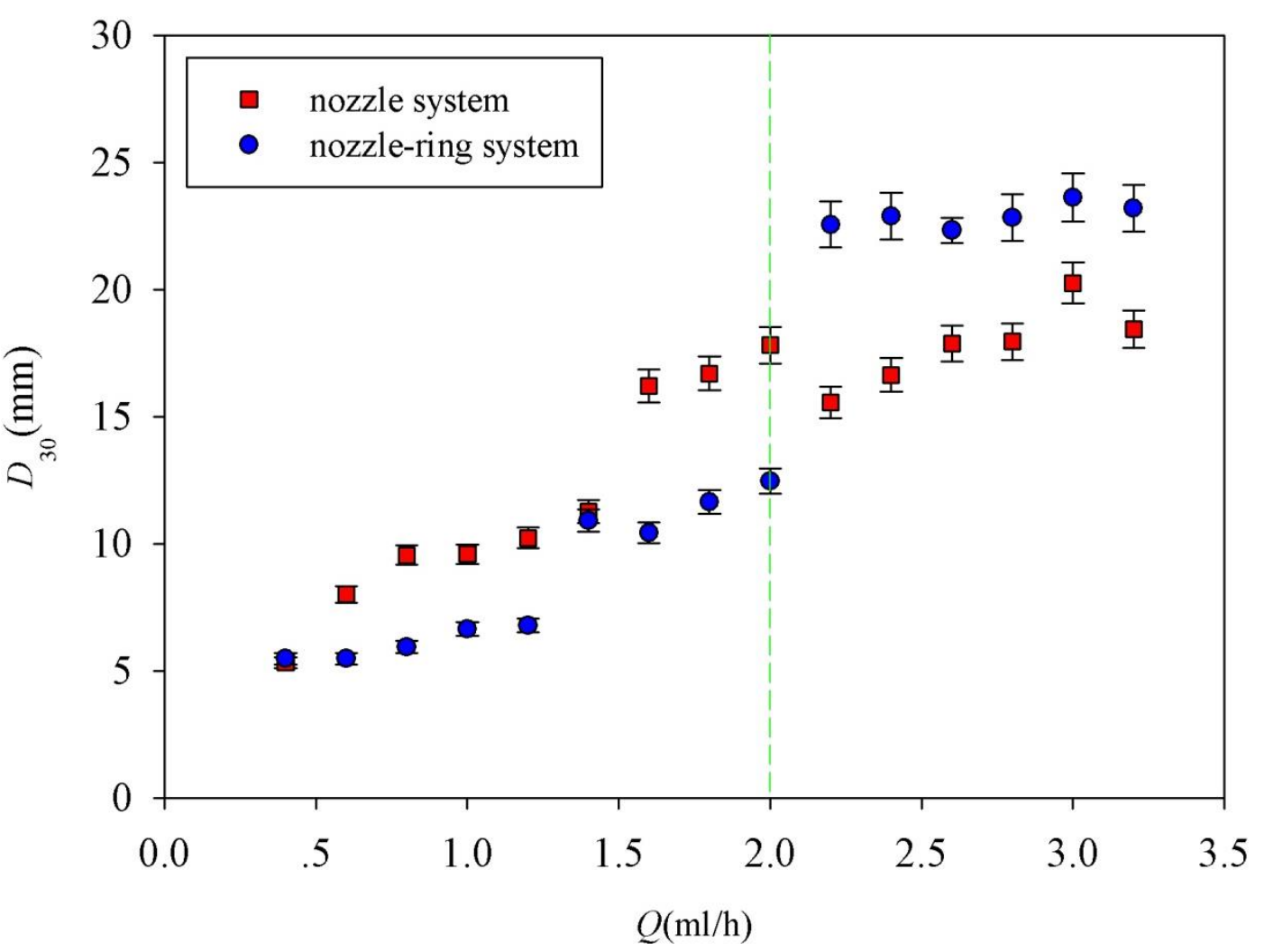

10

11 13

Fig.7 Droplet sizes of ethanol at different flow rates for two electrospray systems at cone-jet mode (Nozzle system: $V_{\mathrm{n}}=5.00-5.50 \mathrm{kV}$; Nozzle-ring system: $V_{\mathrm{n}}=4.00-6.00$

$$
\left.\mathrm{kV}, V_{\mathrm{r}}=1.00 \mathrm{kV}\right)
$$




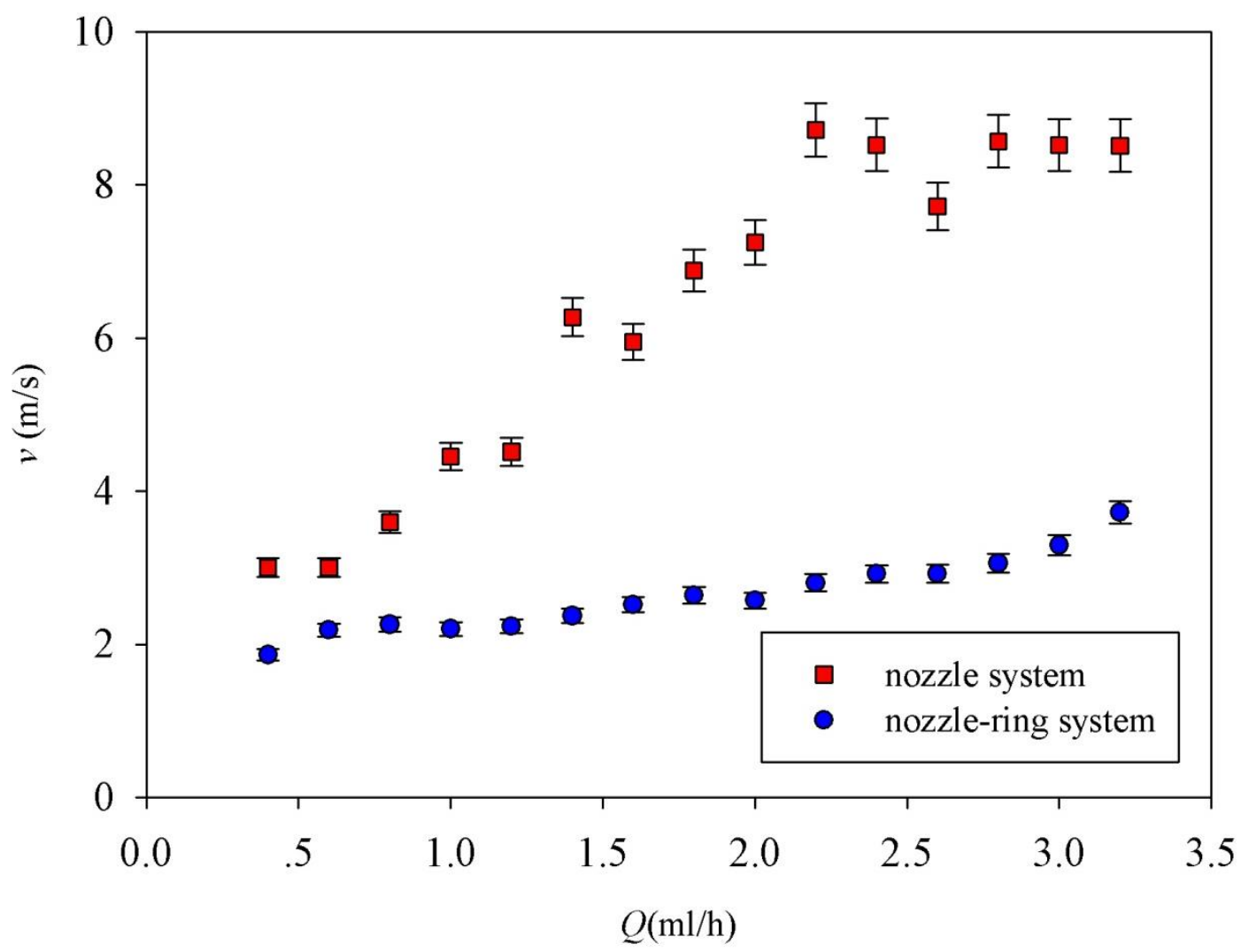

Fig.8 Droplet velocities of ethanol at different flow rates for two electrospray systems at cone-jet mode (Nozzle system: $V_{\mathrm{n}}=5.00-5.50 \mathrm{kV}$; Nozzle-ring system:

$$
\left.V_{\mathrm{n}}=4.00-6.00 \mathrm{kV}, V_{\mathrm{r}}=1.00 \mathrm{kV}\right)
$$

\subsection{The scaling law}

At stable cone-jet mode, a cone-like meniscus is formed at the nozzle tip, from whose apex a jet is emitted. The cone-jet surface is given as the following equation [15]:

$$
r=\xi(z)
$$
where $r$ is the radial coordinate, $z$ is the axial coordinate as shown in Fig. 2, and $\xi$ is the cone-jet surface.

The charge conservation is expressed as [15]:

$$
I=\frac{2 Q \varepsilon_{0}}{\xi} E_{n}+\pi \xi^{2} K E_{s}
$$


1 where $I$ is the total emitted atomization current, the first term on the right-hand side is

2 the induction current, the second term is the conduction current, $Q$ is the flow rate, $\varepsilon_{0}$

3 is the permittivity of a vacuum, $K$ is the electrical conductivity of the liquid, $E_{\mathrm{n}}$ is the

4 normal electric field, and $E_{\mathrm{s}}$ is the tangential electric field.

5 The atomization current $I$ depends on the liquid properties (density $\rho$ and

6 viscosity $\mu$, electrical conductivity $K$, gas-liquid surface tension $\sigma$, and relative

7 permittivity $\varepsilon_{\mathrm{r}}$ ) as well as the flow rate $Q$, nozzle voltage $V_{\mathrm{n}}$, ring voltage $V_{\mathrm{r}}$, vacuum

8 permittivity $\varepsilon_{0}$, and a certain geometrical configuration. For the electro-spraying at the

9 cone-jet mode, the atomization current keeps nearly constant as the voltage changes,

10 so the influence of the voltage can be negligible. The atomization current $I$ is

11 proportional to the square root of the flow rate at cone-jet mode. The characteristic

12 flow rate $Q_{0}$ and current $I_{0}$ are defined as follows [24]:

$$
Q_{0}=\frac{\sigma \varepsilon_{0}}{\rho K}
$$

$$
I_{0}=\left(\frac{\varepsilon_{0} \sigma^{2}}{\rho}\right)^{2}
$$

$$
\frac{I}{I_{0}}=k\left(\frac{Q}{Q_{0}}\right)^{1 / 2}
$$

where $Q_{0}$ is the characteristic flow rate, $I_{0}$ is the characteristic current, and $k$ is a nearly universal constant.

The scaling law indicated by Eq. (8) shows the relationship between the dimensionless current $I / I_{0}$ and the dimensionless flow rate $Q / Q_{0}$.

Fig.9 shows the dimensionless atomization current $I / I_{0}$ as a function of square root of the dimensionless flow rate $Q / Q_{0}$ based on the present experimental results. 

relative error (MRE), defined as

$$
M E R=\frac{1}{M} \sum \frac{\left|I_{\text {pred }}-I_{\text {exp }}\right|}{I_{\text {exp }}} \times 100 \%
$$

4 where $M$ is the number of data points, $I_{\text {pred }}$ is the atomization current predicted by the

5 available correlations, $I_{\exp }$ is the atomization current measured in the experiments.

6 Based on the present experimental results, the fitted equation (10) has a MRE of 2.8\%,

7 and the fitted equation (11) has a MRE of $2.1 \%$.

8

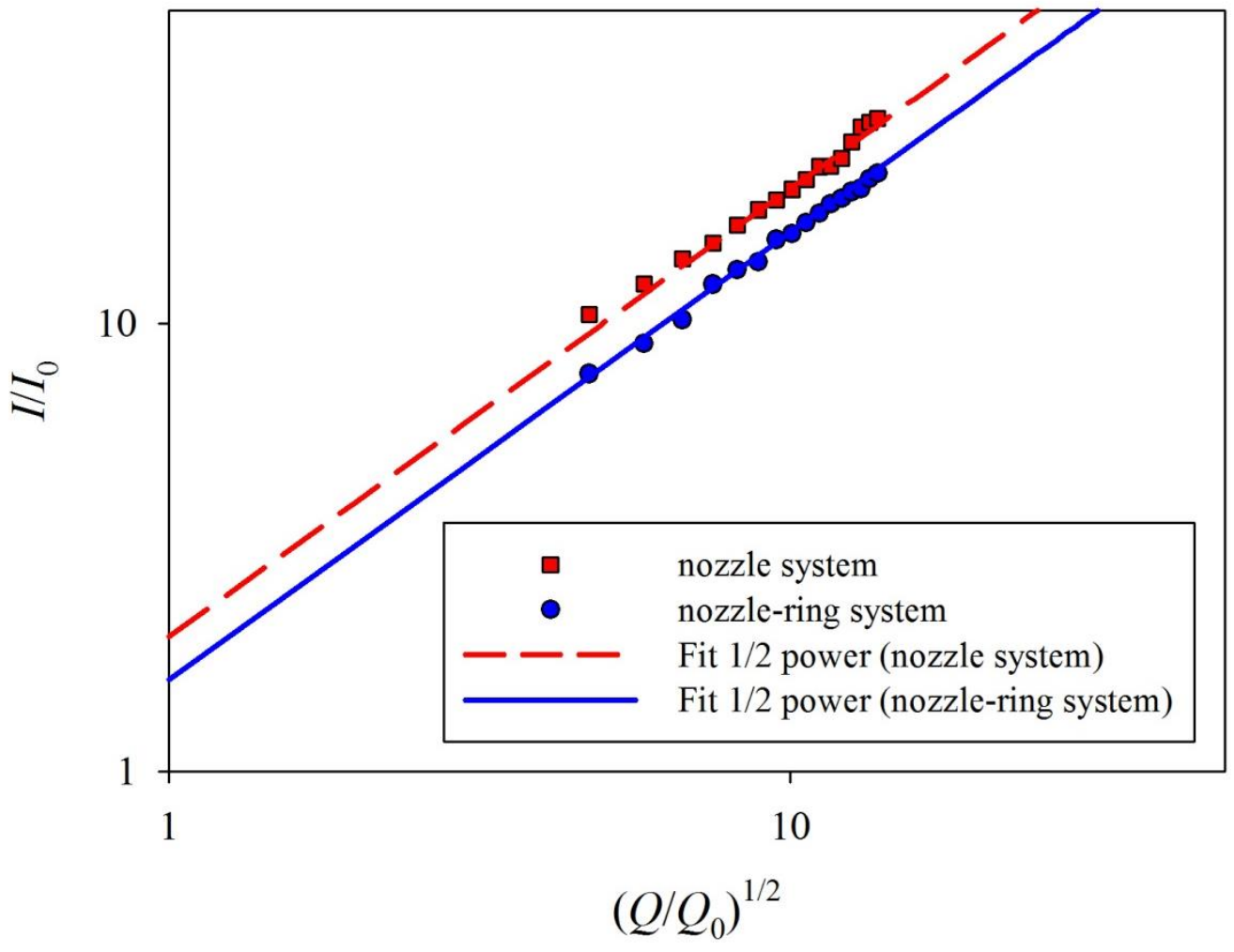

Fig.9 Dimensionless atomization current versus square root of the dimensionless flow rate at cone-jet mode 
$1 \quad$ For the nozzle system:

2

$$
\frac{I_{\text {pred }}}{I_{0}}=2\left(\frac{Q}{Q_{0}}\right)^{1 / 2}
$$

3 For the nozzle-ring system:

$$
\frac{I_{\text {pred }}}{I_{0}}=1.6\left(\frac{Q}{Q_{0}}\right)^{1 / 2}
$$

\section{$5 \quad 3.4$ The relationship between charge density and droplet size}

7 surface tension force. According to the Rayleigh instability, the maximum charge it

8 can sustain is when the electric field force is nearly balanced by the surface tension

9 force, and the limit is called the Rayleigh limit [26],

$$
q=2 \pi \sqrt{2 \varepsilon_{0} \sigma D_{30}^{3}}
$$

where $q$ is the Rayleigh limit charge.

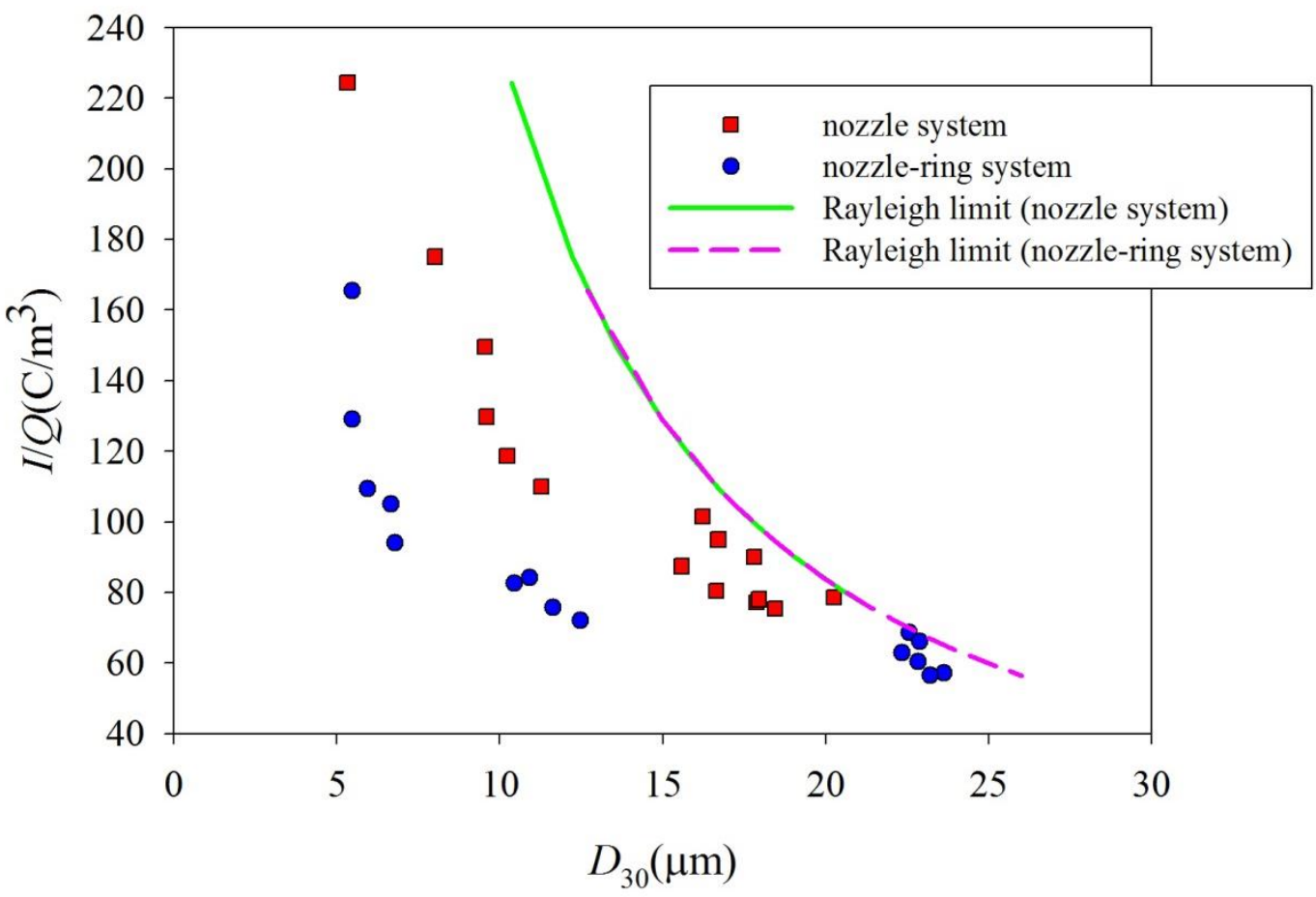



divided by the droplet volume. In order to identify the influence of charge density on

3 the droplet size in the experiments, the charge density can be expressed as the ratio of

4 the atomization current to the liquid flow rate. The maximum charge density is 5 defined as

$$
\frac{q}{V}=\frac{I}{Q}=\frac{12 \sqrt{2 \varepsilon_{0} \sigma}}{D_{30}^{3 / 2}}
$$
where $V$ is the droplet volume. It indicates that charge density have a -1.5 power dependence on droplet diameter. And the smaller droplet has higher charge density. that both the nozzle system and the nozzle-ring system show a good agreement with the Rayleigh instability.

\section{Conclusions} combustors are carried out using liquid ethanol as fuel. The electro-spraying systems are the same with that used in our previous work [19]. The electro-spraying systems consist of the nozzle system (without a ring electrode) and the nozzle-ring system (with a ring electrode). The photos of electro-spraying at cone-jet mode are taken by a digital camera. The voltage drop across the resistance between the grid and the ground is measured by a data acquisition instrument, and the atomization current is calculated according to Ohm's law. The size and velocity distributions of electro-spraying droplets are measured by a Phase Doppler Anemometer. A non-dimensional analysis 
1 on atomization current has been proposed to explain the electro-spraying phenomena

2 of liquid at the stable cone-jet mode. The following can be concluded:

3 (1) At cone-jet mode, the smaller atomization currents and droplet velocities

4 corresponds to the smaller droplet sizes.

5 (2) The dimensionless atomization current in both nozzle system and nozzle-ring

6 system obey the scaling law as square root of the dimensionless flow rate.

7 (3) The charge density has a -1.5 power dependence on the droplet diameter; both the

8 nozzle system and the nozzle-ring system show a good agreement with the Rayleigh

9 instability.

\section{Acknowledgements} 22

The authors gratefully acknowledge the National Nature Science Foundation of China (51376066, 51611130194), Project of Guangzhou Science and Technology Plan (201707010071), State Key Laboratory of Engines, Tianjin University (K2016-01).

\section{列}

\section{8} 9

\section{0} 1 


\section{References}

[1] Fernandez-Pello, A. C. (2002). Micropower generation using combustion: Issues and approaches. Proceedings of the Combustion Institute, 29(1):883-898.

[2] Gan, Y. H., Luo, Y. L., Wang, M., Shi, Y. L., \& Yan Y. Y. (2015a). Effect of alternating electric fields on the behaviour of small-scale laminar diffusion flames. Applied Thermal Engineering, 89:306-315.

[3] Zeleny, J.(1917). Instability of electrified liquid surfaces. Physical Review, 10 (1): $1-6$.

[4] Jaworek, A., Sobczyk, A., Czech, T., \& Krupa, A. (2014).Corona discharge in electrospraying. Journal of Electrostatics, 72:166-178.

[5] Wang, H.C., \& V.Mamishev, A. (2012). Optimization methodology for electrospray evaporative cooling chambers. Journal of Electrostatics, 70:384-392.

[6] Jaworek, A., \& Sobczyk, A.T. (2008). Electrospraying route to nanotechnology: An overview. Journal of Electrostatics, 66: 197-219.

[7] Ku, B. K. \& Kim, S. S. (2003). Electrohydrodynamic spraying characteristics of glycerol solutions in vacuum. Journal of Electrostatics, 57:109-128.

[8] Lastow, O. \& Balachandran, W. (2006). Numerical simulation of electrohydrodynamic (EHD) atomization. Journal of Electrostatics, 64:850-859.

[9] Lastow, O. \& Balachandran, W. (2007).Novel low voltage EHD spray nozzle for atomization of waterin the cone jet mode. Journal of Electrostatics, 65:490-499.

[10] Agathou, M. (2010). Biobutanol fuel atomization and combustion processes.

Ph.D. thesis, University of Illinois at Urbana-Champaign, 3-4. 
1 [11] Thong, K. C. \& Weinberg, F. J. (1971). Electrical Control of the Combustion of

2 Solid and Liquid Particulate Suspensions. Proceedings of the Royal Society of

3 London. Series A, Mathematical and Physical Sciences, 324:201-215.

4 [12] Gañán-Calvo, A. M., Davila, J. \& Barrero, A. (1997). Current and droplet size in

5 the electrospraying of liquids. Scaling laws. Journal of Aerosol Science, $6 \quad 28(2): 249-275$.

7 [13] Fernandez de la Mora, J. \& Loscertales, I. G. (1994). Current emitted by highly

8 conducting Taylor cones. Journal of Fluid Mechanics, 260:155-184.

9 [14] Gañán-Calvo, A. M., Lasheras, J. C., Davila, J. \& Barrero, A. (1994).The emitted current and droplet size laws in steady cone-jet electrosprays of polar and non-polar 11 liquids. Journal of Aerosol Science, 25:1121-1142.

[15] Gañán-Calvo, A. M. (2004). On the general scaling theory for electrospraying.

Journal of Fluid Mechanics, 507: 203-212a.

[16] Holbrook, L., Hindle, M. \& Longest, P. W. (2015). Generating charged pharmaceutical aerosols intended to improve targeted drug delivery in ventilated infants. Journal of Aerosol Science, 88:35-47.

[17] Li, C., Chang, M., Yang, W. W., Madden, A. \& Deng, W. W. (2014). Ballpoint pen tips as robust cone-jet electrospray emitters. Journal of Aerosol Science, 77:10-15.

[18] Gan, Y. H., Luo, Z. B., Cheng, Y. P. \& Xu, J. L. (2015b). The electro-spraying characteristics of ethanol for application in a small-scale combustor under combined electric field. Applied Thermal Engineering, 87:595-604. 
1 [19] Gan, Y. H., Zhang, X., Li, H. G., Tong, Y., Zhang, Y., Shi, Y. L. \& Yang, Z. L.

2 (2016). Effect of a ring electrode on the cone-jet characteristics of ethanol in

3 small-scale electro-spraying combustors. Journal of Aerosol Science, 98:15-29.

4 [20] Paine, M. D., Alexander, M. S. \& Stark, J. P. W. (2007). Nozzle and liquid effects

5 on the spray modes in nanoelectrospray. Journal of Colloid and Interface Science,

$6 \quad 305: 111-123$.

7 [21] Alexander, M. S. (2008). Pulsating electrospray modes at the liquid-liquid

8 interface. Applied Physics Letters, 92: (144102).

9 [22] Verdoold, S., Agostinho, L. L. F., Yurteri, C. U. \& Marijnissen, J. C. M. (2014). A generic electrospray classification. Journal of Aerosol Science, 67:87-103.

11 [23] Yaws, C.L. (2009). Chemical Properties Handbook, McGraw-Hill.

12 [24] Higuera, F. J. (2004). Current/flow-rate characteristic of an electrospray with a 13 small meniscus. Journal of Fluid Mechanics, 513:239-246.

14 [25] Hartman, R. P. A. (1998), Electrohydrodynamic atomization in the cone-jet mode, 15 Ph.D. thesis, Technische Universiteit Delft. [26] Strutt J. W., Rayleigh, L. (1883), Investigation of the character of the equilibrium 17 of an incompressible heavy fluid of variable density. Proc. London Math. Soc., 14: $170-177$. 